ISSN 1997-5902

\title{
Évaluation de la production en fruits de peuplements naturels de Baobab (Adansonia digitata L.) dans deux zones climatiques au Sénégal
}

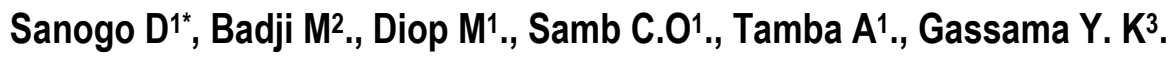 \\ $1=$ ISRA-Centre national de recherche forestière (CNRF) BP 2312 Dakar (Sénégal) \\ 2= Laboratoire d'écologie végétale et d'écohydrologie (UCAD/FST) BP 5005 Dakar (Sénégal) \\ 3= Université Cheikh Anta Diop, département de biologie végétale BP 5005 Dakar (Sénégal) \\ * Diaminatou SANOGO ISRA/CNRF, Bp 2312 Dakar Sénégal, Email : sdiami@yahoo.fr
}

Original submitted in on 28th October 2014. Published online at www.m.elewa.org on 30th January 2015 http://dx.doi.org/10.4314/jab.v85i1.8

\section{RÉSUMÉ}

Objectif : Ce travail vise à évaluer la production en fruits et en pulpe de A.digitata afin de mieux connaître ses potentialités dans deux zones climatiques au Sénégal.

Méthodologie et résultats : L'évaluation de la production a porté sur 88 et 54 arbres. Les poids des fruits et de la pulpe ont été étudiés par rapport à des descripteurs physiques de l'arbre. Des équations de régression entre ces variables ont été établies. La production moyenne par arbre est $35,5 \mathrm{~kg}$ de fruits en zone soudano-sahélienne contre $64,9 \mathrm{~kg}$ de fruits en zone soudano guinéenne. La moyenne de $46,7 \mathrm{Kg}$ de fruits par arbre correspond à une production de pulpe de $28,28 \mathrm{~kg}$ par arbre. II existe des régressions significatives entre les paramètres allométriques de l'arbre et les caractères de productivité.

Conclusion et application : L'espèce Adansonia digitata a de grandes potentialités fruitières dans les deux zones climatiques prospectées. A l'issue de ces travaux, il est possible de prédire le potentiel de production d'un baobab à partir des données morphométriques de l'arbre. Les équations de régression établies constituent des outils performants qui peuvent être utilisés par les techniciens forestiers pour évaluer la production des formations naturelles et des parcs agroforestiers, voire la productivité des individus dans le cadre d'une domestication. Ils pourraient également aider les décideurs locaux et les industriels à la prise de décision dans le contexte de la décentralisation et de développement d'entreprises privées. Aussi, la variabilité de la fructification de Adansonia digitata pourra être exploitée par les futurs programmes de sélection et d'amélioration génétique.

Mots clés : Sénégal, Adansonia digitata, production, fruits, zone climatique

\section{Évaluation of Baobab (Adansonia digitata $L$.) fruit production in Senegal ABSTRACT}

Objective: This work aims to evaluate the fruit and pulp production of A.digitata in order to know its potential in two climate zones in Senegal.

Methodology and results: The evaluation of the production covered 88 and 54 trees. The fruit and pulp weight were studied compared with tree physical descriptors. Regression equations between these variables were established. The average production per tree is $35.5 \mathrm{~kg}$ of fruit In the Sudano-Sahelian zone against $64.9 \mathrm{~kg}$ of fruit in Sudano guinean zone. The average of $46.7 \mathrm{~kg}$ of fruit per tree corresponds to a pulp production of $28.28 \mathrm{~kg}$ per tree. There are significant regressions between the Allometric parameters of the tree and productivity traits. 
Conclusion and application: Adansonia digitata species has a great potential fruit in two climatic zones surveyed. Through this work, it is possible to predict the production potential of a baobab from morphometric data of the tree. The regression equations developed are powerful tools that can be used by foresters to evaluate the production of natural formations and parklands and in addition the productivity of individuals in a domestication context. They also could help local makers and industrials in the decision-making in the decentralization and private enterprises development contexts. In addition, the Adansonia digitata fruiting variability can be exploited by the future breeding programs and genetic improvement.

Key words: Sénégal, Adansonia digitata, production, fruits, climatic zone

\section{INTRODUCTION}

Au Sahel, le baobab (Adansonia digitata L.) est une espèce à usages multiples très estimée par les populations (Collière, 2001, Bationo et al 2010). Au Sénégal, il fait partie de la liste des espèces prioritaires (Soloviev et al., 2004 ; Faye et al., 2010). L'espèce contribue de façon significative à la subsistance en milieu rural et à l'économie des populations locales et nationale (Codjia et al., 2001 ; Sidibé et Williams, 2002 ; Cissé et al., 2005 ; Assogbadjo et al., 2006, Sanogo et Tamba, 2012). L'autorisation, ces dernières années, de l'Union européenne d'incorporer la pulpe du fruit de baobab dans les boissons a donné une valeur économique accrue à l'espèce à l'échelle internationale. Depuis 2009, annuellement, plus de 100 tonnes de pulpe brute et 50 tonnes de graines de baobab sont respectivement transformés en poudre et huile exportées par des industries agroalimentaires comme «Baobab Fruit Company " et "Bioessence » (Sanogo et Tamba, 2012). Les graines, les fibres et coques de fruits du baobab sont également utilisées

\section{MATÉRIEL ET MÉTHODES}

Localisation de l'étude: Une évaluation de la production en fruits de $A$. digitata a été réalisée en 2012 sur des populations de parcs à baobab du Sénégal, localisées dans les communes rurales de Bala (zone soudano-sahélienne) et de Dar Salam (zone soudano-guinéenne) (Figure 1) ; elles ont été choisies en fonction de l'existence des parcs à baobab (figure 2), l'importance de la production fruitière et de la demande des programmes et industries qui y interviennent. Ces deux zones se distinguent l'une de l'autre par leurs conditions climatiques et biophysiques. La commune rurale de Bala se situe dans la région de Tambacounda au sud-est du Sénégal entre la latitude $14^{\circ} 02^{\prime}$ Nord et longitude $13^{\circ} 08^{\prime}$ Ouest. Le climat de cette zone est comme composantes de produits « $\mathrm{BIO}$ » dans l'industrie et l'agriculture. En dépit de cette importance avérée des produits issus du baobab en général et de sa pulpe en particulier, on ne dispose que de peu d'informations sur son potentiel de production en termes de rendement en fruits bruts. Les quelques travaux menés dans ce domaine portent sur l'estimation de la production des capsules de baobab dans les différentes zones climatiques du Bénin (Assogbadjo et al., 2005). Les études effectuées jusqu'à présent sur le baobab au Sénégal, ne renseignent pas sur le potentiel de production de l'espèce dans les zones de production. De ce fait, l'objectif de notre étude est d'évaluer la production en fruits et en pulpe de A.digitata dans deux zones climatiques au Sénégal. Les données ainsi collectées pourront être utiles aux décideurs locaux et aux industriels qui veulent savoir quelles quantités de fruits de Adansonia digitata peuvent être attendues des parcs à baobab du Sénégal.

de type soudano-sahélien avec une pluviométrie variant entre 600 et $800 \mathrm{~mm}$. La moyenne pluviométrique des dix dernières années est estimée à $724 \mathrm{~mm}$ pour 55 jours moyens de pluie par an. Le type de végétation dominant est la savane arbustive et boisée des plateaux et pénéplaines qui sont surtout des zones de culture. On y rencontre: Adansonia digitata, Bombax Costatum, Borassus aethiopium Combretum glutinosum, Combretum nigricans, Cordyla pinnata, Pterocarpus erinaceus, Prosopis africana, Sterculia setigera, et Ziziphus mauritiana. En revanche, la commune rurale de Dar Salam se situe dans la région de Kédougou à l'extrême sud-est du Sénégal entre la latitude $12^{\circ} 63^{\prime}$ Nord et la longitude $12^{\circ} 78^{\prime}$ Ouest. Le climat de cette 
zone est de type soudano-guinéen. L'indice pluviométrique connait une variabilité interannuelle, mais elle se situe généralement entre 1000 et 1200 $\mathrm{mm}$. La moyenne pluviométrique annuelle pour les dix dernières années est estimée à $1197 \mathrm{~mm}$. Le type de végétation dominant est la savane boisée et par endroit on rencontre la forêt dense sèche et des mosaïques de forêts galeries. On y rencontre des espèces guinéennes telles que Antiaris africana, Ceiba pentandra, Daniellia oliveri, Khaya senegalensis, Spondias mombin et des espèces soudaniennes comme Borassus flabellifer, Cordyla pinnata, Oxytenanthera abyssinica, Pterocarpus erinaceus et Vitellaria paradoxa.

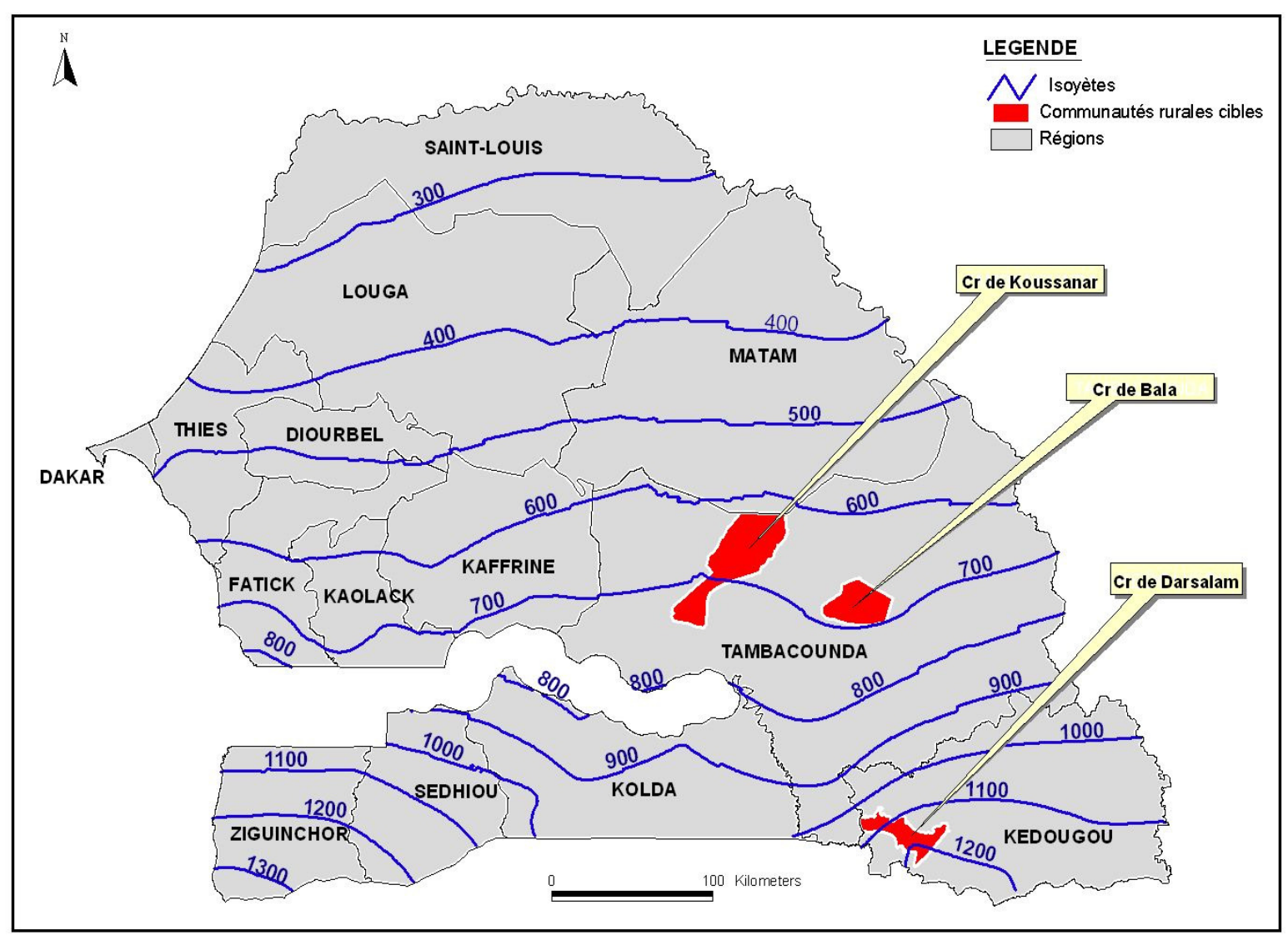

Figure 1 : Localisation des zones d'étude de la production des baobabs, limites des zones climatiques et principales villes du Sénégal (source CSE 2012 source CSE 2012)

Échantillonnage: L'évaluation a porté sur un échantillon de 142 individus pris au hasard parmi 742 arbres qui avaient été inventoriés dans le cadre d'une étude de la structure démographique de $A$. digitata, à raison de 88 arbres dans la zone soudano-sahélienne (Bala) et 54 dans la zone soudano-guinéenne (Dar salam). Notre choix a porté sur des individus de toutes les classes de diamètre qui se trouvaient en pleine production des fruits. Les échantillons de Bala (zone soudano sahélienne) et de Dar Salam (zone soudano- guinéenne) étaient respectivement composés de :

- $\quad 26$ et 9 individus de classe de diamètre inférieure ou égale à $1 \mathrm{~m}$,

- $\quad 45$ et 33 individus de classe de diamètre comprise entre 1 et $2 \mathrm{~m}$

17 et 12 individus de classe de diamètre supérieure ou égale à $2 \mathrm{~m}$ 

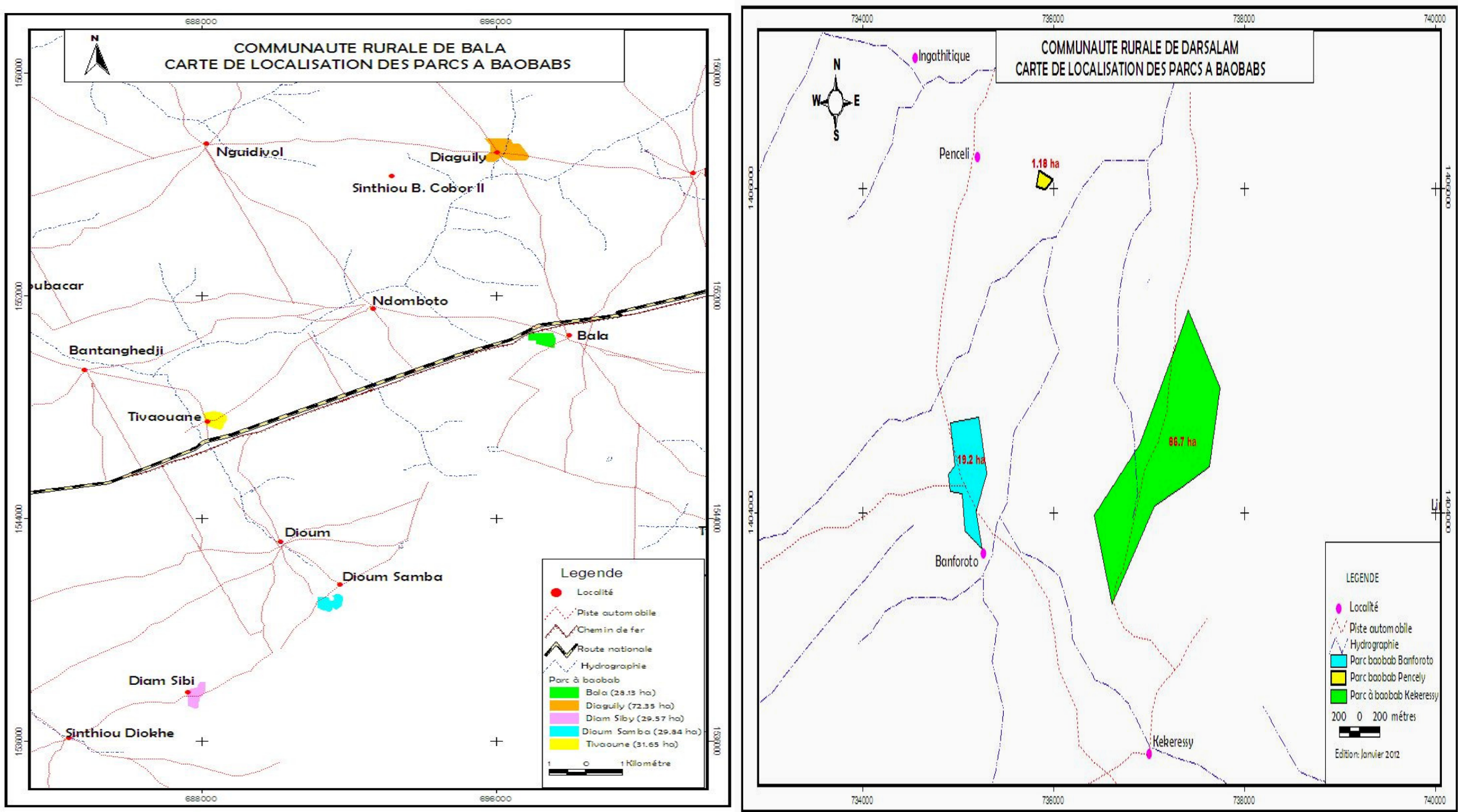

Figure 2 : Localisation des parcs de Baobabs dans les communautés rurales de Bala (a) et Dar Salam (b) au Sénégal 
Paramètres mesurés sur les arbres: Les mensurations sur l'architecture des arbres ont porté sur les caractères suivants : le diamètre du tronc à $1,30 \mathrm{~m}$ du sol, la hauteur de l'arbre et le diamètre du houppier, le nombre de fruits. Ces données permettent de voir le lien qui existe entre la morphologie des arbres et les caractères de la production.

Évaluation de la production en fruits et en pulpe : Pour l'évaluation de la production fruitière, d'une manière générale, les fruits ont été intégralement cueillis à l'aide d'une perche crochue longue de 3 à 4 mètres sur des individus numérotés, ensuite ils ont été ramassés, comptés et pesés. Dans les cas où la récolte intégrale était impossible en raison de l'inaccessibilité de certains fruits ou de leur état d'immaturité, ils ont été comptés et leur poids a été estimé et rapporté à la quantité finale. Sur la base d'enquêtes de terrain, il a pu être vérifié que, pour un même individu, la forme des capsules de baobab est relativement stable (Assogbadjo et al 2005). Pour l'évaluation de la production en pulpe, un échantillon de $30 \%$ du nombre total de fruits a été pesé et placé dans des sacs en jute étiquetés et emportés. Pour un arbre donné, le nombre total des fruits d'une part et le poids moyen des fruits et de la pulpe d'autre

\section{RÉSULTATS}

Production fruitière de Adansonia digitata : La production fruitière par individu de $A$. digitata a été en moyenne de $35,5 \mathrm{~kg}$ de fruits en zone soudanosahélienne (Bala), et de $64,9 \mathrm{~kg}$ de fruits en zone soudano guinéenne (Dar Salam) (Tableau 1). II en résulte que le rendement moyen en fruits des parcs de $A$. digitata en zone soudano-sahélienne et en zone soudano-guinéenne pourrait potentiellement être respectivement $468,6 \mathrm{~kg} \mathrm{ha}^{-1}$ et $558,14 \mathrm{~kg} \mathrm{ha}^{-1}$. Cependant la densité des baobabs dans la zone soudano-sahélienne est plus élevée (13,2 arbre ha- $\left.^{-1}\right)$ que dans la zone soudano-guinéenne ( 8,6 arbre ha1). La production moyenne en pulpe par individu a été de $23,4 \mathrm{~kg} \mathrm{ha}^{-1}$ par arbre en zone soudano- part étant connu, il a été possible de déterminer, la production en fruit et en pulpe d'un arbre.

Traitement et analyse des données : Les analyses statistiques des résultats ont été effectuées avec le logiciel XLSTAT version 2010. Les données de la circonférence ont été transformées en diamètre sur la base de l'équation : circonférence $=\pi x$ diamètre . Le diamètre moyen du houppier a été calculé comme la moyenne des deux mesures de la circonférence (nord-sud et est-ouest). La production de fruits et de pulpe par arbre a été estimée en utilisant le nombre total de fruits et le poids moyen des fruits ou poids moyen de la pulpe. La production de fruits par ha a été estimée en utilisant la production moyenne d'un arbre et la densité des parcs de Adansonia digitata estimé à 13,2 $\pm 10,71$ et 8,6 $\pm 2,54$ arbres ha- 1 respectivement à Bala et Dar salam dans une étude antérieure (Sanogo et Tamba, 2012). Des analyses descriptives ont été faites pour voir la dispersion de la production de Adansonia digitata dans les deux différentes zones climatiques. Ensuite des corrélations et des régressions linéaires entre les descripteurs physiques (diamètre du tronc à $1,30 \mathrm{~m}$ du sol, hauteur de l'arbre et diamètre du houppier) et les paramètres de productivité (nombre total des fruits, poids total des fruits, poids total de la pulpe) ont été établies avec le test Fisher.

sahélienne et de 36,69 kg ha-1 en zone soudanoguinéenne.

Corrélation : La matrice des corrélations permettant d'étudier les descripteurs de la production fruitière de A. digitata montre que le diamètre du tronc à $1,30 \mathrm{~m}$ du sol est positivement et significativement corrélé au nombre des fruits $(r=0,50)$, au poids des fruits ( $r$ $=0,48)$ et au poids de pulpe $(r=0,49)$ et que le nombre et le poids des fruits sont positivement et significativement corrélés entre eux $(r=0,84)$ (Tableau 2).

Ces valeurs de coefficient $r$ permettent de déduire qu'au sein des populations de baobab au Sénégal, les arbres bons producteurs sont de gros diamètre. 
Tableau 1. Caractéristiques biométriques de la production fruitière d'Adansonia digitata au Sénégal (mesures effectuées sur la production de 88 arbres à Bala, 54 à Dar Salam et 142 pour les deux zones)

\begin{tabular}{|c|c|c|c|c|c|c|c|}
\hline \multicolumn{2}{|c|}{ Production fruitière } & $\begin{array}{c}\text { Diamètre tronc à } \\
1,3 \mathrm{~m}(\mathrm{~m})\end{array}$ & $\begin{array}{c}\text { Diamètre } \\
\text { houppier }(\mathrm{m})\end{array}$ & Hauteur (m) & $\begin{array}{l}\text { Nombre de } \\
\text { fruits }\end{array}$ & $\begin{array}{l}\text { Poids fruits } \\
\text { larbre (kg) }\end{array}$ & $\begin{array}{l}\text { Poids pulpe /arbre } \\
(\mathrm{kg})\end{array}$ \\
\hline \multirow{3}{*}{$\begin{array}{c}\text { Zone soudano sahélienne } \\
\text { Bala (88 arbres) }\end{array}$} & Moyenne & 1,38 & 17,69 & 26,81 & 133,03 & 35,52 & 23,4 \\
\hline & Minimum & 0,48 & 4,5 & 13 & 7 & 1,6 & 0,96 \\
\hline & Maximum & 3,2 & 28,9 & 39,4 & 646 & 338,38 & 150,4 \\
\hline \multirow{3}{*}{$\begin{array}{l}\text { Zone soudano guinéenne } \\
\text { Dar Salam (54 arbres) }\end{array}$} & Moyenne & 1,4 & 15,69 & 24,58 & 218,04 & 64,93 & 36,69 \\
\hline & Minimum & 0,5 & 7,75 & 13 & 4 & 1,73 & 1,47 \\
\hline & Maximum & 2,4 & 24 & 39,4 & 1217 & 244,01 & 102,27 \\
\hline \multirow{3}{*}{$\begin{array}{c}\text { Toutes les zones (142 } \\
\text { arbres) }\end{array}$} & Moyenne & 1,39 & 16,93 & 24,57 & 203,90 & 46,7 & 28,28 \\
\hline & Minimum & 0,48 & 4,5 & 13 & 4 & 1,6 & 0,96 \\
\hline & Maximum & 3,2 & 28,9 & 39,4 & 1217 & 338,38 & 150,4 \\
\hline
\end{tabular}


Sanogo et al.. J. Appl. Biosci. Évaluation de la production en fruits de peuplements naturels de Baobab (Adansonia digitata L.) dans deux zones climatiques au Sénégal)

Régression: Les équations de régressions calculées à partir de ces mêmes descripteurs de la production fruitière de $A$. digitata montrent qu'il existe une régression significative d'une part entre le poids des fruits et le diamètre du tronc à $1,30 \mathrm{~m}$ du sol et entre le poids de pulpe et le diamètre du tronc à 1,30 $\mathrm{m}$ du sol, d'autre part entre le nombre de fruits et le diamètre du tronc à $1,30 \mathrm{~m}$ du sol (Tableau 3 , figure 3 , figure 4). En revanche, la régression n'est pas significative entre le nombre de fruits et le diamètre moyen du houppier (Tableau 3). Les droites de régression (figures 3 et 4 ) indiquent un faible coefficient de détermination, bien que les régressions soient hautement significatives.

Tableau 2: Corrélation entre les descripteurs de la production fruitière de Baobab mesurés sur un échantillon de 142 arbres étudiés au Sénégal

\begin{tabular}{|l|c|c|c|c|c|c|}
\hline & $\begin{array}{c}\text { Diamètre } \\
\text { à 1,30 } \mathrm{m}\end{array}$ & $\begin{array}{c}\text { Hauteur } \\
\text { de l'arbre }\end{array}$ & $\begin{array}{c}\text { Diamètre } \\
\text { moyen du } \\
\text { houppier }\end{array}$ & $\begin{array}{c}\text { Nombre } \\
\text { total de } \\
\text { fruits }\end{array}$ & $\begin{array}{c}\text { Poids de } \\
\text { fruits/arbre }\end{array}$ & $\begin{array}{c}\text { poids de } \\
\text { pulpe/arbre }\end{array}$ \\
\hline $\begin{array}{l}\text { Diamètre à 1,30 } \mathrm{m} \\
\text { du sol }\end{array}$ & 1 & 0,640 & 0,735 & 0,504 & 0,489 & 0,491 \\
\hline Hauteur de l'arbre & 0,640 & 1 & 0,796 & 0,198 & 0,268 & 0,310 \\
\hline $\begin{array}{l}\text { Diamètre moyen du } \\
\text { houppier }\end{array}$ & 0,735 & 0,796 & 1 & 0,359 & 0,386 & 0,406 \\
\hline $\begin{array}{l}\text { Nombre total de } \\
\text { fruits }\end{array}$ & 0,504 & 0,198 & 0,359 & 1 & 0,848 & 0,723 \\
\hline Poids de fruits/arbre & 0,489 & 0,268 & 0,386 & 0,848 & 1 & 0,874 \\
\hline Poids de pulpe/arbre & 0,491 & 0,310 & 0,406 & 0,723 & 0,874 & 1 \\
\hline
\end{tabular}

Tableau 3 : Régression linéaire entre le diamètre du tronc pris à $1,30 \mathrm{~m}$ du sol, le nombre de fruits et certains descripteurs de la production pour un échantillon de 142 arbres de $A$ dansonia digitata au Sénégal

\begin{tabular}{|l|c|c|c|}
\hline \multicolumn{1}{|c|}{ Équation de régression } & $\begin{array}{c}\text { Coefficient de } \\
\text { détermination }\end{array}$ & Probabilité & Signification \\
\hline Poids de fruits = 42,467 Diam 1,30m - 12,35 & 0,240 & $<0,0001$ & ${ }^{* * *}$ \\
\hline Poids pulpe = 23,211 Diam 1,30m - 3,9431 & 0,243 & $<0,0001$ & ${ }^{* * *}$ \\
\hline Nombre de fruits = 82,528 Diam 1,30m - 20,376 & 0,259 & 0,0001 & ${ }^{* * *}$ \\
\hline Poids de fruits = 3,1377Diammoyhoup -6,714 & 0,149 & $<0,0001$ & ${ }^{* * *}$ \\
\hline poids pulpe = 1,7849Diammoyhoup - 1,9383 & 0,164 & $<0,0001$ & ${ }^{* * *}$ \\
\hline Nombre de fruits = 2,0534Diammoyhoup +59,447 & 0,004 & 0,44 & Ns \\
\hline
\end{tabular}

${ }^{* * *}$ Hautement significatif au seuil de $5 \%$., ns : non significatif., Diam 1,30m : diamètre du tronc à $1,30 \mathrm{~m}$ du sol , Diammoyhoup : diamètre moyen du houppier $(\mathrm{m})$. 

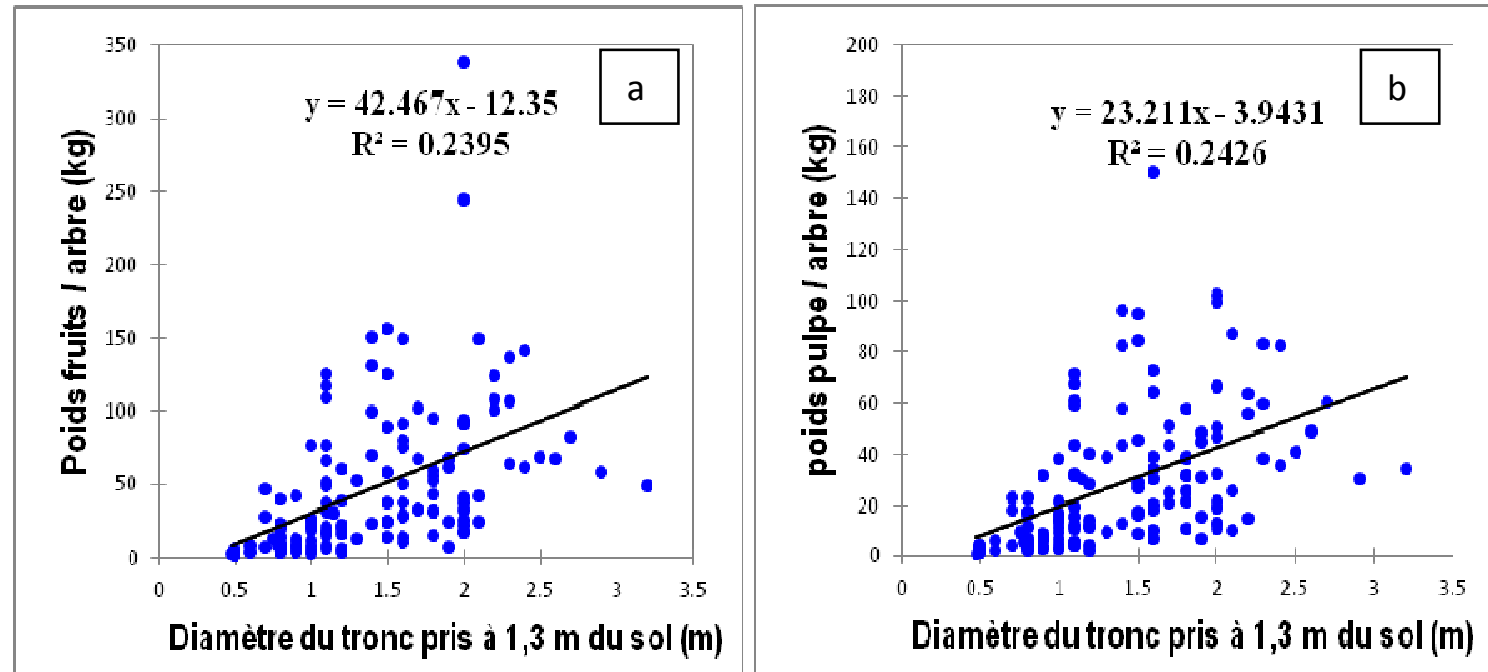

Figure 3 : Relations entre la production de fruits/arbre et le diamètre du tronc à $1,30 \mathrm{~m}$ du sol (a) et entre la production de pulpe par /arbre (b) et le diamètre du tronc à 1,30 m du sol de Adansonia digitata (échantillonnage de 142 arbres observés au Sénégal).

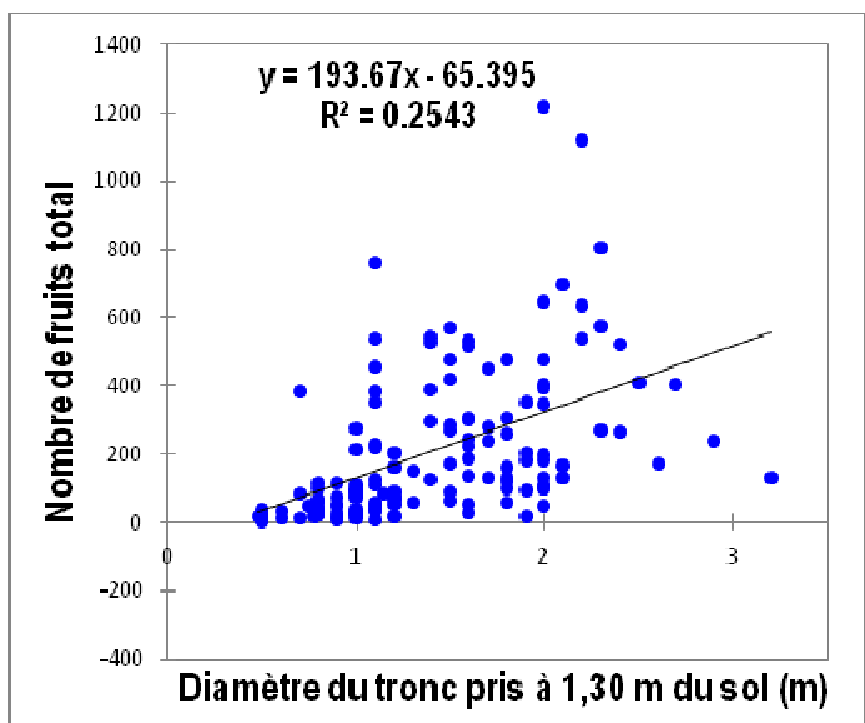

Figure 4 : Relation entre le nombre de fruits /arbre et le diamètre du tronc à $1,30 \mathrm{~m}$ du sol de Adansonia digitata (échantillonnage de 142 arbres observés au Sénégal).

\section{DISCUSSION}

Notre étude sur l'évaluation de la production en fruits et en pulpe de baobab dans deux zones climatiques au Sénégal est la première qui ait été entreprise sur ce thème dans ce pays. L'approche quantitative utilisée a permis la mise en évidence d'une relation entre les paramètres allométriques de l'arbre et ses caractères de productivité. L'étude montre qu'en zone soudano-sahélienne, la production moyenne par arbre est de $35,5 \mathrm{~kg}$ de fruits entre les extrêmes de 1,6 et 338,38 contre $64,9 \mathrm{~kg}$ de fruits entre les extrêmes de $1,73 \mathrm{~kg}$ et $244,01 \mathrm{~kg}$ en zone soudano guinéenne. La variation de la quantité de fruits produits par arbre est donc très marquée selon les écarts type 42,28 et 55, 01 autour des moyennes. En effet cette forte variation met en évidence la grande variabilité qui caractérise la production du baobab d'un sujet à un autre. Des études menées au Benin sur la caractérisation de la variabilité morphologique et de production (Assogbajo et al, 2005) et celles sur la diversité morpho-biochimique des morphotypes paysans de baobab au Sénégal (Sanogo et al 2013) ont confirmé cette variabilité qui était principalement liée aux conditions d'environnement et d'habitat; selon Assogbadjo et al 2005, cette variabilité pourrait probablement être liée à une diversité génétique existante au sein des individus de baobab. Par 
ailleurs, des irrégularités de fructification analogues ont également été observées chez Detarium microcarpum au Mali (Kouyaté et al., 2006) et Vitellaria paradoxa au Burkina Faso (Guira et Zongo, 2002). A défaut de littérature assez fournie sur la production fruitière de $A$. digitata, nous avons ramené et comparé nos résultats avec ceux des études portant sur la production des capsules de baobab au Benin. En effet, le poids moyen d'un fruit de baobab pour les 54 sujets que nous avons étudié à Dar Salam (zone Soudano-guinéenne) est de $224,34 \mathrm{~g}$, valeur proche de la moyenne de $273 \mathrm{~g}$ obtenu au Benin dans les conditions climatiques presque similaires (Assogbadjo et al., 2005). Par ailleurs, les meilleurs rendements en fruits et en pulpe de baobab ont été obtenus dans la zone soudano-guinéenne, ces résultats sont également conformes à ceux de Assogbadjo et al. (2005) qui signalaient que cette zone climatique était intéressante en termes de productivité du baobab. .

\section{CONCLUSION}

L'évaluation de la production fruitière de Adansonia digitata a permis de mettre en évidence les potentialités fruitières de cette espèce dans deux zones climatiques au Sénégal. A l'issue de ces travaux, il est possible de prédire le potentiel de production d'un baobab à partir des données morphométriques de l'arbre. Les équations de régression établies constituent des outils performants qui peuvent être utilisés par les

\section{RÉFÉRENCES BIBLIOGRAPHIQUES}

Assogbadjo A E, Kyndt T, Sinsin B, Gheysen G, Van Damme P, 2006. Patterns of genetic and morphometric diversity in Baobab (Adansonia digitata), Populations accross different climatic zones of Benin (West Africa). Annals of Botany $97: 819-830$.

Assogbadjo A E, Sinsin B, Van Damme P, 2005. Caractères morphologiques et production des capsules de baobab (Adanasnia digitata L.) au Bénin. Fruits 60 (5) : 327340.

Bationo A B, Maïga A, Compaore $P$, Kalinganire A, 2010. Dimension socioculturelle du baobab Adansonia digitata L. dans le Plateau central du Burkina Faso. Bois et Forêts des Tropiques, $N^{\circ} 306$ (4) : 23-32.

Boffa J M, 2000.West African agroforestry parklands: key to conservation and sustainable management. Unasylva 51 (200): 11-17.
La corrélation significative et positive entre le diamètre du tronc à $1,30 \mathrm{~m}$ du sol et la production fruitière et de pulpe de $A$. digitata corroborent avec les résultats d'autres travaux réalisés sur des espèces fruitières forestières soudano-sahéliennes (Le Houerou, 1980 ; Breman et Kessler, 1995, Kouyaté et al 2006). Ces corrélations permettent de dire que dans notre population de baobab, les arbres bons producteurs sont de gros diamètre. Ce constat est en accord avec les conclusions de Boffa (2000) et Lamien et al. (2008) qui soutiennent que le nombre et le poids moyen des fruits de Vitellaria paradoxa étaient positivement corrélés à la taille de l'arbre. Par contre, au Burkina Faso, Guira et Zongo (2002) ont trouvé une corrélation négative entre le diamètre et la production de Vitellaria paradoxa indiquant que les arbres bons producteurs sont de petite taille.

techniciens forestiers pour évaluer la production des formations naturelles et des parcs agroforestiers, voire la productivité des individus dans le cadre d'une domestication. Ils pourraient également aider les décideurs locaux et les industriels à la prise de décision. Aussi, la variabilité de la fructification de Adansonia digitata pourra être exploitée par les futurs programmes de sélection et d'amélioration génétique.

Breman H, Kessler J J, 1995. Woody plants in agroecosystems of semi-arid regions with emphasis on the Sahelian countries, Springer-Verlag. Adv. Ser. Agric. Sci. 23, Berlin, Germany. $340 \mathrm{p}$.

Cissé M, Sakho M, Dornier M, Mar Diop C, Reynes $\mathrm{M}$, Sock O, 2005. Caractérisation du fruit du baobab et étude de sa transformation en nectar. Fruits, vol.16 : 19-34.

Codjia J T C, Fonton B K, Assogbadjo A E, Ekue M R M, 2001. Le baobab (Adansonia digitata). Une espèce à usage multiple au Bénin. CECODI/CBDD/VeCo/SNV/FSA, Benin, $44 p$.

Collière $\mathrm{P}, 2001$. Étude de la forêt de Baobabs (Adansonia digitata L.) de Ngékoh (Sénégal): analyse des causes de la dégradation de la forêt et recherches de solutions. Rapport d'étude associations chênes et baobabs. 34p. 
Faye M D, Weber J C, Abasse T A, Boureima M, Larwanou M, Bationo A B, Diallo B, Sigué H, Dakouo J M, Samaké O, Sanogo D, 2010. Farmers' preferences for tree functions and species in the West african sahel. Forests, Trees and Livelihoods, Vol. 20: $113-136$.

Guira M et Zongo J D, 2002. Évaluation de la production d'une population de karité, Vitellaria paradoxa (Gaertn.f) (Sapotaceae) au Burkina Faso. Bulletin de la Recherche Agronomique $N^{\circ} 38$ : 16-25.

Kouyaté AM, Van Damme $P, 2006$. Caractères morphologiques de Detarium microcarpum Guill. et Perr. au sud du Mali, Fruits 57 : 231-238.

Lamien N, Some A N, Ouédraogo J S, llboudo B, Ouédraogo C, Somba I, 2008. Potentiel productif de quelques essences fruitières locales au Burkina Faso. Science et Technique, sciences naturelles et agronomie, Vol. 30, n¹: 61-73.

Le Houerou HN, 1980. Techniques agroforestières pour la conservation et l'amélioration de la fertilité des sols dans les zones arides et semi-arides. In : Le Houerou H.N. (Éd.), Les fourrages ligneux en Afrique. État actuel des connaissances. Colloq. int. fourrag. Ligneux Afr., CIPEA, Addis Ababa, Éthiopie : 421-424.

Sanogo D et Tamba A, 2012. Inventaire des Parcs de Baobab et du potentiel pain de singe dans les CR de Koussanar et Bala dans la Région de Tambacounda et la CR de Dar Salam dans la Région de Kédougou. Rapport final d'étude MEA-USAI, Programme Agriculture Gestion des Ressources Naturelles «Wula Nafa» Sénégal 52p.

Sanogo D. Diedhiou B., Diop A., Sanou H., Thiome M. Gassama Y K, et Bassène E., 2013. Morphologic and Biochemical Diversity of Peasant's Baobab Tree (Adansonia digitata L.) Morphotypes in Senegal, African Natural Plant Products Volume II: Discoveries and Challenges in Chemistry, Health, and Nutrition; H. Rodolfo Juliani., James E. Simon., and Chi-Tang Ho. ACS Symposium Series; American Chemical Society: Washington, DC, 16: 256-271

Sidibé M, Williams J T, 2002. Baobab (Adansonia digitata). International center for underutilised crops, Southampton, UK.
Soloviev P, Niang TD, Gaye A, Totte A, 2004. Variabilité des caractères physicochimiques des fruits de trois espèces ligneuses de cueillette, récoltés au Sénégal : Adansonia digitata, Balanites aegyptiaca et Tamarindus indica, Fruits $59: 109-119$. 